\title{
BLICKDIAGNOSE-QUIZ
}

\author{
Nur ein Nebenbefund
}

\section{Ein schöner Rücken kann auch entzücken}

\section{Auflösung von Seite 5}

— Es handelt sich bei diesen Hautveränderungen um ausgeprägte seborrhoische Keratosen, auch Alterswarzen genannt. Die seborrhoische Keratose ist der häufigste benigne Tumor der Haut. Fast alle Menschen entwickeln im Alter unabhängig von exogenen Einflüssen seborrhoische Keratosen. Sie können an fast sämtlichen Stellen der Haut auftreten, bevorzugt im Gesicht, an den Handrücken und am Oberkörper. Fußsohle und Handinnenfläche werden ausgespart.

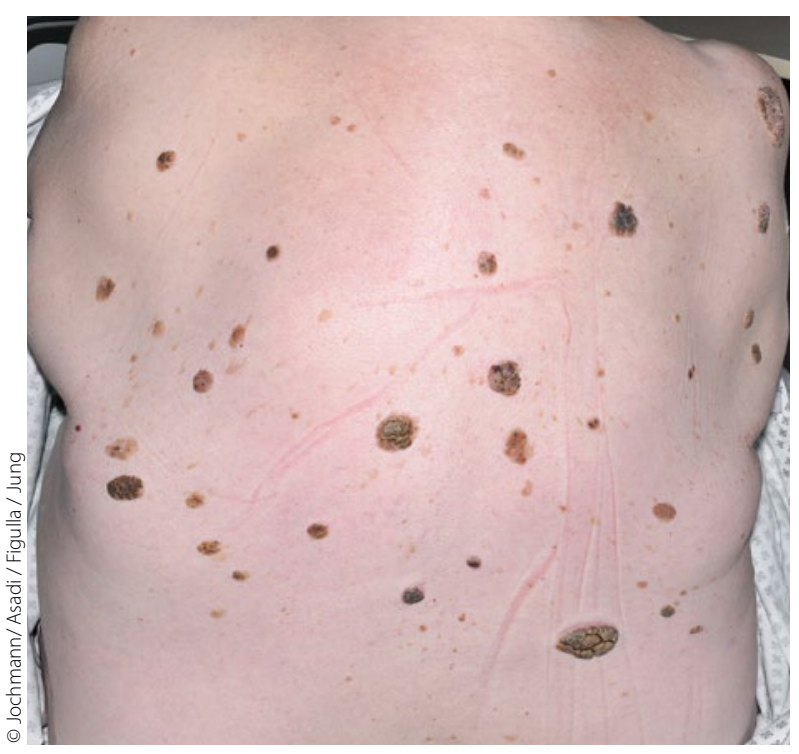

\section{Ausgeprägte seborrhoische Keratose.}

Die symptomlosen, harmlosen Tumoren variieren stark in ihrer Morphologie. Sie entstehen über Jahre hinweg, sind zunächst flach, scharf begrenzt, zunehmend hyperpigmentiert und breiten sich horizontal aus. Sie können sich endophytisch weiter- entwickeln (Lentigo senilis) oder aber exophytisch auswölben (seborrhoische Keratose). Seborrhoische Keratosen sind in der Regel im Durchmesser bis zu einem Zentimeter groß, können aber auch bedeutend größere Ausmaße annehmen. Sie fühlen sich fettig an - daher auch der Name.

Die Diagnose ergibt sich aus dem klinischen Bild und dem zeitlichen Verlauf. Bei unklaren Befunden sollte eine histopathologische Untersuchung erfolgen.

Differenzialdiagnostisch ist das Leser-Trélat-Syndrom in Betracht zu ziehen. Hierbei handelt es sich um eine von einigen Autoren als paraneoplastisches Syndrom bezeichnete Hautveränderung, die durch das plötzliche Auftreten vieler, in etwa 50\% der Fälle stark juckender, seborrhoischer Keratosen gekennzeichnet ist und meist in Kombination mit gastrointestinalen Tumoren auftritt.

Da es sich bei seborrhoischen Keratosen um benigne Tumoren ohne Potenzial zur malignen Entartung handelt, wird keine Therapie empfohlen. Stark störende Befunde, die gegebenenfalls mit mechanischer Irritation, Blutung und Infektionsneigung einhergehen, können mittels Kürettage mit elektrischer Schlinge oder scharfem Löffel in Lokalanästhesie entfernt werden.

Keywords: senile warts

- Elisabeth Jochmann, Dr. med. Yahya Asadi, Prof. Dr. med. Hans-R. Figulla, Dr. med. Christian Jung, Klinik für Innere Medizin I der Universitätsklinik Jena, Erlanger Allee 101, D-07747 Jena 